\title{
Simulation of the performance of organic solar cells based on D1-BT-EDOT-BT-D2-A/PCBM structures
}

\author{
Imane EL Mhamedi ${ }^{1, *}$, Anass El Karkri ${ }^{1, *}$, and Zakaria El Malki ${ }^{1}$ \\ ${ }^{1}$ Moulay Ismail University, Research team: Modeling, Materials and Systems Control (MMSC). Research laboratory: Computer \\ Engineering and Intelligent Electrical Systems (2ISEI). High School of Technology Meknes (ESTM) BP: 3103, Toulal, Meknes, \\ Morocco
}

\begin{abstract}
The analys is of microelectronic and photonic structures-one dimension (AMPS-1D) was used to study and simulate the performance of organic heterojunction solar cells based on D1 -BEdot-B-D2-A as electron donors, and [6,6]-phenyl-C61-butyric acid methyl ester (PCBM) as an electron acceptor. The organic photovoltaic cell devices using T3-B-Edot-B-T3-A/PCBM showed the improved open-circuit voltage Voc, short-circuit current density Jsc, fill factor FF, and power conversion efficiency PCE values for the optimum thickness of $120 \mathrm{~nm}$ and the effective state density of electrons and holes of $10^{21} \mathrm{~cm}^{-3}$.The P3-B-Edot-B-T3-AVPCBM and P3-B-Edot-B-P3AVPCBM based devices exhibited a power conversion efficiency (PCE) of $9.295 \%$ and $8.735 \%$, respectively, which outperformed the corresponding T3-B-Edot-B-T3-AVPCBM, Cbz-B-Edot-B-T3APCBM, F-B-Edot-B-T3-A/PCBM, and A-B-Edot-B-T3-A/PCBM based devices (7.330, 6.622, 7.226, and 7.327\%). More importantly, the P3-B-Edot-B-T3-A/PCBM and P3-B-Edot-B-P3AVCBM -based device delivered the highest PCE of $14.432 \%$ and $15.031 \%$ respectively, when we deposit a layer of PEDOT between the indium tin oxide (ITO) and the active layer, which is a clear improvement over other results in the literature.
\end{abstract}

\section{Introduction}

The reference scenario of the international energy agency (IEA) forecasts that global primary energy needs will increase by $55 \%$ between 2005 and 2030, due in particular to exponential growth in emerging countries [1]. Faced with this ever-growing demand and the exhaustion of fossil fuels (oil, gas, coal), which currently remain the majority primary sources of electricity production in the world $(60 \%)$ [2], the use of alternative energies has become a priority. Renewable energies produced by a natural process and which are inexhaustible and do not emit no greenhouse gases, unlike fossil fuels [3]. The value of the power conversion efficiency (PCE) for organic solar cells remains lower if compared to the efficiency obtained by other technologies (Silicium 25\% [4]), however, several reasons justify the efforts made to develop the organic sector, and which lie in the advantages of organic materials. Indeed, this technology would allow access to flexible photovoltaic modules and/or large surfaces. Molecular engineering also enables adaptation of band gap values and energy levels of organic materials, which are relatively easy to produce at low cost $[5,6]$. In recent years, the power conversion efficiencies (PCEs) of organic solar cells have been rapidly improved with the development of photoactive layers containing higher performing acceptors and donors [7,18]. Electronic conduction in the case of organic materials requires the possibility of creation or injection of free charge carriers. For molecules, this implies easy ionization; either by ejection of electrons form HOMOS in the molecule (Highest Occupied Molecular Orbitals), or by electron capture in LUMOs (Lowest Unoccupied Molecular Orbitals). This extraction or injection is particularly easy in the case of compounds having a delocalized $\pi$ electron system [19]. These compounds are characterized by the regular alternation of single and double bonds allowing delocalization of electrons along the backbone. It is the conjugation phenomenon. In this work, the AMPS-1D program was used to study and simulate the performance of organic solar cells, based on organic matter consisting of an acceptor materiel: [6,6 Acid methyl ester]-phenylC61-butyric acid (PCBM) [20], and a donor material: D1B-EDOT-B-D2-A (composed of benzothiadiazole (B), 3,4-Ethylenedioxythiophene (EDOT), the cyanoacrylic acid anchoring group (A), and different donor unit consisting of thiophene (T), Phenylene (P), Carbazole (C), Fluorene (F) and Antrhacene (A)). The geometric, electrical, molecular, and optical properties of the molecules used in this article [21] were investigated using the density functional theory and time dependent density Functional Theory (DFT and TD-DFT) / B3LYP / 6$31 \mathrm{G}(\mathrm{d}, \mathrm{p})$ method. The main objective of this work is to define the influence of thickness of the active layer, temperature, the effective state density of electrons and holes, and the effect of changing the unit donor on the electrical characteristic of the organic photovoltaic cell and subsequently on its parameters. The second objective is to study the influence of the addition of a PEDOT layer between the anode (ITO) and the active layer on the performance of the photovoltaic solar cell.

\footnotetext{
${ }^{1}$ Corresponding author: i.elmhamedi@edu.umi.ac.ma; a.elkarkri@edu.umi.ac.ma
} 


\section{Parameters of organic solar cells}

The plot of the current-voltage characteristic provides access to many physical parameters of the component. The first parameters that appear on the current-voltage characteristic of a photovoltaic cell are the short-circuit current density (Jsc), the open-circuit voltage (Vco), the fill factor (FF) of the component, and the power conversion efficiency (PCE).

\subsection{The short-circuit current density (Jsc)}

This is the most important current density that can be obtained with a solar cell. It grows linearly with the illumination intensity of the cell and depends on the illuminated area, the wavelength of the radiation, the mobility of the carriers and the temperature [22].

\subsection{The open-circuit voltage (Vco)}

In the case of organic solar cells, the Vco is linearly dependent on the level of the HOMO of the donor material and on the level of the LUMO of the acceptor material $[23,24]$. In addition, the charge losses at the materialelectrode interfaces may also affect the value of the Vco [25].

The theoretical values of open-circuit voltage $V_{\text {ocwere }}$ calculated from the following expression [25]:

$$
V_{\text {OC }}=\left|E_{\text {HOMO }}^{\text {Donor }}\right|-\left|E_{\text {LUMO }}^{\text {Acceptor }}\right|-0.3
$$

\subsection{The fill factor FF}

The fill factor FF reflects the degree of ideality of the J(V) curves. It is determined by the following equation:

$$
\mathrm{FF}=\frac{\mathrm{Pmax}}{\mathrm{Voc} \cdot \mathrm{Jsc}}=\mathrm{Vmax} \cdot \frac{\mathrm{Jmax}}{\mathrm{Voc} \cdot \mathrm{Jsc}}
$$

Where Jmax and Vmax respectively represent the current density and voltage of the operating point that allows to extract the maximum power (Pmax) of the cell. The fill factor can also provide information on the quality of the material-electrode interfaces.

\subsection{The power conversion efficiency}

The power conversion efficiency $\eta$ of photovoltaic cells is defined as the ratio between the maximum power Pmax delivered by the cell and the incident light power, Pin. [26]

$$
\eta=\frac{F F \cdot V_{O C} \cdot J_{S C}}{P_{i n}}
$$

The maximum conversion efficiency of a cell is only meaningful for a given spectral distribution and intensity. The most commonly used standard irradiation corresponds to an air mass number AM1.5. PCEs in the range of $6-10 \%$ were achieved in recent years for a single BHJ solar cell [27, 32] and a PCE of $10.1 \%$ [33] and 12\% for tandem cell devices [34].

\section{The studied molecules}

In this work, we will study and simulate the photovoltaic properties of organic solar cells based on the D1-BEDOT-B-D2-A oligomers (figure 1).

The optimized structure and the geometric properties calculated by B3LYP / 6-31 (d, p) show that donors and acceptors are fully linked through the $\pi$-conjugated bridges whose are all planar.

These molecules present a law band gap, and there LUMO energy levels are higher than that of PCBM $(-3,9 \mathrm{eV})$ (figure 2).

This condition is sufficiently important for efficient electron injection. HOMO and LUMO analysis of the oligomers indicates the electron or hole transport capability.

This condition is sufficiently important for efficient electron injection. HOMO and LUMO analysis of the oligomers indicates the electron or hole transport capability.

It is clearly seen that the electron density of the highest occupied molecular orbitals (HOMO) is mainly localized on the donor part of (3,4-Ethylenedioxythiophene) and diffusely distributed on the $\pi$-conjugation on the Benzothiadiazole ring. In contrast, the electron density of the lowest unoccupied molecular orbitals (LUMO) is extended over the acceptor acid groups (figure 2).

The capacity of the material to accept one electron from the environment is the electronic affinity EA.

$$
\mathrm{EA}=\mathrm{E}(\mathrm{M})-\mathrm{E}\left(\mathrm{M}^{-}\right)
$$

Where, $\mathrm{E}(\mathrm{M})$ and $\mathrm{E}\left(\mathrm{M}^{-}\right)$are the total energies of neutral molecule and anion states, respectively. We calculated the energies using the B3LYP/6-31G(d,p) level.

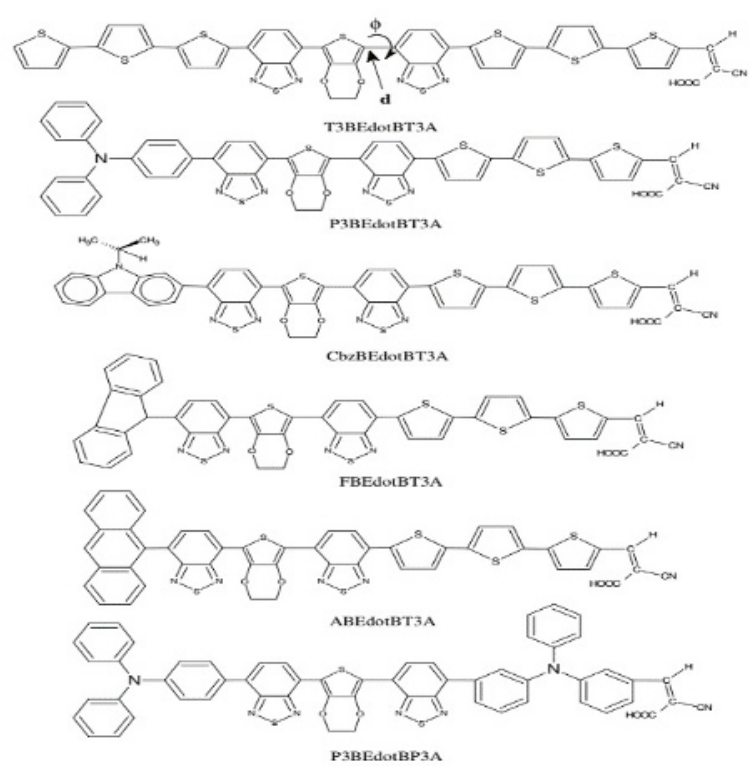

Fig. 1. The sketch map structures of studied organic oligomers [21]. 


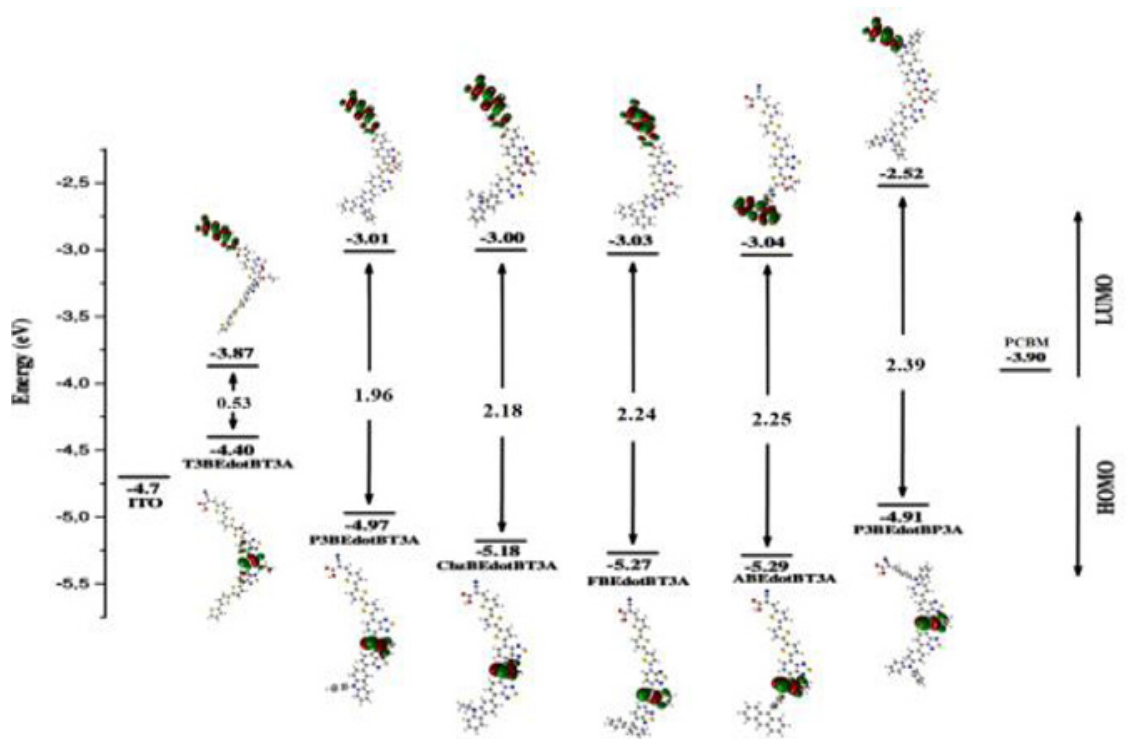

Fig. 2. The sketch of B3LYP/6-31G(d,p) calculated energies of the HOMO and LUMO level and the contour plots of HOMO and LUMO orbitals of the studied molecules [21].

A good photovoltaic material should have broad and strong visible absorption characteristics. The simulated absorption spectra of these oligomers are shown in Figure 3 The $\lambda_{\max }$ values of these oligomers are in excellent agreement with $E_{\text {gap }}$ values previously presented.

Table 1. The EA $(\mathrm{eV})$ of the studied molecules obtained by B3LYP/6-31G (d,p) level [21].

\begin{tabular}{|c|c|c|c|}
\hline compounds & EA(eV) & compounds & EA(eV) \\
\hline T3BEdotBT3A & 3.35 & FBEdotBT3A & 2.22 \\
\hline P3BEdotBT3A & 2.23 & ABEdotBT3A & 2.22 \\
\hline CbzBEdotBT3A & 2.21 & P3BEdotBP3A & 1.78 \\
\hline
\end{tabular}

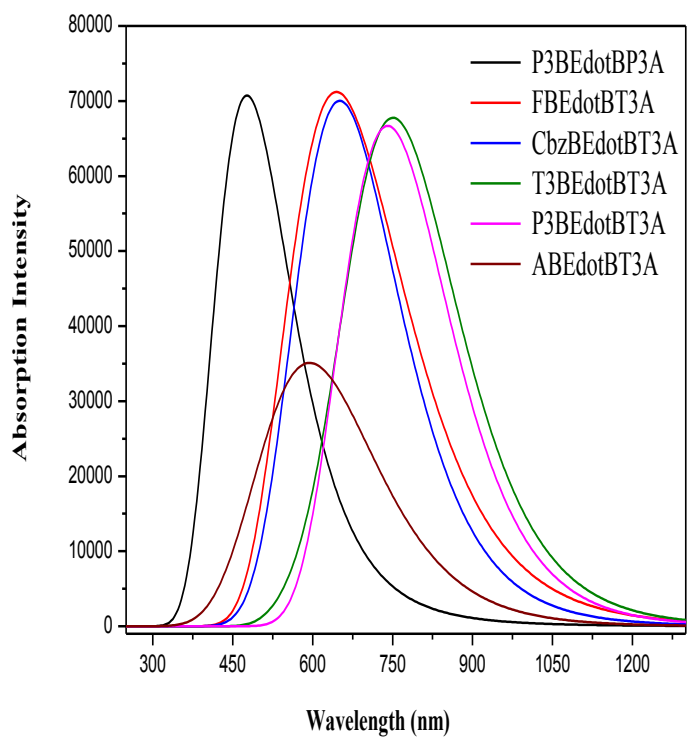

Fig. 3. Simulated UV-Visible optical absorption spectra of the studied compounds calculated by TD/DFT/B3LYP/6-31G (d, p) level [21].

\section{Results and discussion}

\subsection{Studied Structure}

In this part of the work, we will focus our efforts on the characterization of an ITO/T3-B-EDOT-B-T3-A/ $\mathrm{PCBM} / \mathrm{Al}$ type organic photovoltaic cell as it is shown in Figure 4.

$[6,6]$-phenyl-C61-butyric acid methyl ester (PCBM) is a derivative of Buckminster's C60 fullerene, it has been widely used in combination with $\mathrm{P} 3 \mathrm{HT}$ and other donors in organic solar cells due to its high electron mobility properties $[35,36]$ and its ability to organize into a structure favorable for good electronic conductivity [37].

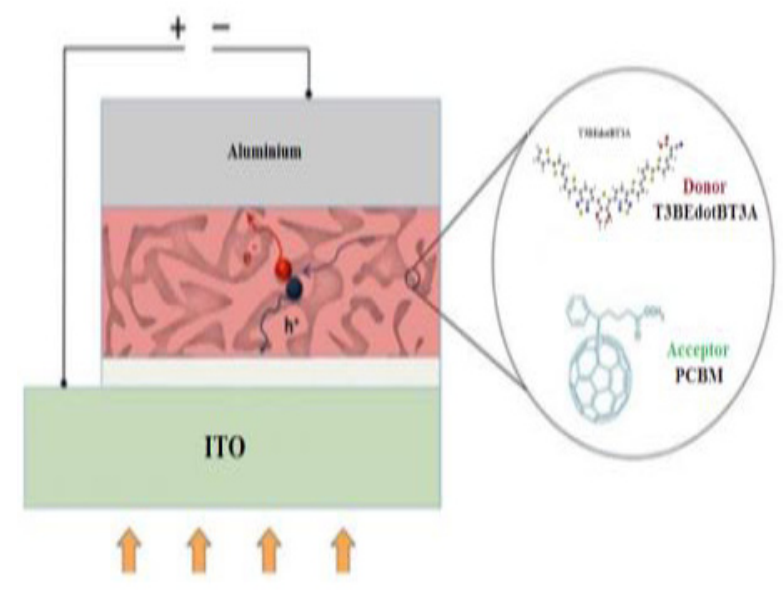

Fig. 4. Bulk heterojunction structure of the organic solar cell based on T3BEdotBT3A / PCBM.

\subsection{Influence of the Thickness}

For a fixed temperature $(300 \mathrm{~K})$, we will study the impact of the active layer thickness on the properties of the organic solar cell based on T3-B-EDOT-B-T3-A/ PCBM (Figure 5). From the obtained results, we deduce that the conversion efficiency values increase with the increase of 
the active layer thickness; this is essentially due to a good absorption of the thicker layers, since the light absorption is one of the determining factors of the photovoltaic efficiency. With the increase of the thickness of the active layer, we notice that the short-circuit current density Jsc increases, the open-circuit voltage Voc remains constant, while the fill factor FF decreases slightly from $100 \mathrm{~nm}$. On the other hand, if we compare our results obtained by simulation with those obtained in the case of $\mathrm{P} 3 \mathrm{HT} / \mathrm{PCBM}$, we notice an improvement in the performance of the solar cell. Table 2 shows the results obtained by varying the thickness from 80 to $180 \mathrm{~nm}$. The results show that the optimum efficiency for T3BEdotBT3A/PCBM is at $120 \mathrm{~nm}$ thickness because the development of active layers with a great thickness, poses many technical problems, which inevitably generate higher costs. The experimental results give an optimum thickness of about $100 \mathrm{~nm}$ [38].

Table 2.Photovoltaic parameters of the organic cell based on T3-B-EDOT-B-T3-A/ PCBM for different thickness.

\begin{tabular}{|c|c|c|c|c|}
\hline $\begin{array}{c}\text { Thickness } \\
(\mathrm{nm})\end{array}$ & $\begin{array}{c}\mathrm{Jsc}_{\mathrm{sc}}(\mathrm{mA} \\
\mathrm{cm}_{-} 2\end{array}$ & $\mathrm{Voc}(\mathrm{eV})$ & FF & $\eta \%$ \\
\hline 80 & 12.601 & 0.607 & 0.725 & 5.543 \\
\hline 100 & 14.264 & 0.609 & 0.726 & 6.303 \\
\hline 120 & 15.725 & 0.609 & 0.720 & 6.903 \\
\hline 140 & 17.021 & 0.609 & 0.711 & 7.372 \\
\hline 160 & 18.165 & 0.609 & 0.700 & 7.742 \\
\hline 180 & 19.155 & 0.608 & 0.690 & 8.036 \\
\hline
\end{tabular}

\subsection{Effect of the Temperature}

In this section, the temperature dependence of cell parameters was investigated, using the AMPS program and taking $120 \mathrm{~nm}$ as thickness. For a temperature varying between 280 to $320^{\circ} \mathrm{K}$, the results are presented in table 3.The temperature has some influence on the performance of the organic cell. The cell parameters ( $\eta$, Jsc, Voc, FF) decrease with increasing temperature. However, the impact of temperature change on the open circuit voltage Voc in organic cells is significantly lower than that of inorganic cells, where it was found that the open circuit voltage decreases with increasing temperature, implying a significant advantage of organic photovoltaic cells in many fields of use.

Table 3. Photovoltaic parameters of the organic cell based on T3-B-EDOT-B-T3-A/PCBM for different temperatures.

\begin{tabular}{|c|c|c|c|c|}
\hline $\begin{array}{c}\text { Temperature } \\
\left({ }^{\circ} \mathrm{K}\right)\end{array}$ & $\begin{array}{c}\text { Jsc (mA } \\
\left.\mathrm{cm}_{\_}\right)\end{array}$ & Voc(eV) & FF & $\eta \%$ \\
\hline 280 & 15.780 & 0.635 & 0.734 & 7.357 \\
\hline 285 & 15.766 & 0.628 & 0.731 & 7.239 \\
\hline 290 & 15.752 & 0.622 & 0.727 & 7.124 \\
\hline 295 & 15.738 & 0.616 & 0.724 & 7.012 \\
\hline 300 & 15.725 & 0.609 & 0.720 & 6.903 \\
\hline 305 & 15.711 & 0.603 & 0.717 & 6.798 \\
\hline 310 & 15.698 & 0.597 & 0.715 & 6.697 \\
\hline 315 & 15.686 & 0.591 & 0.712 & 6.598 \\
\hline 320 & 15.671 & 0.585 & 0.709 & 6.501 \\
\hline & & & &
\end{tabular}

Light IV

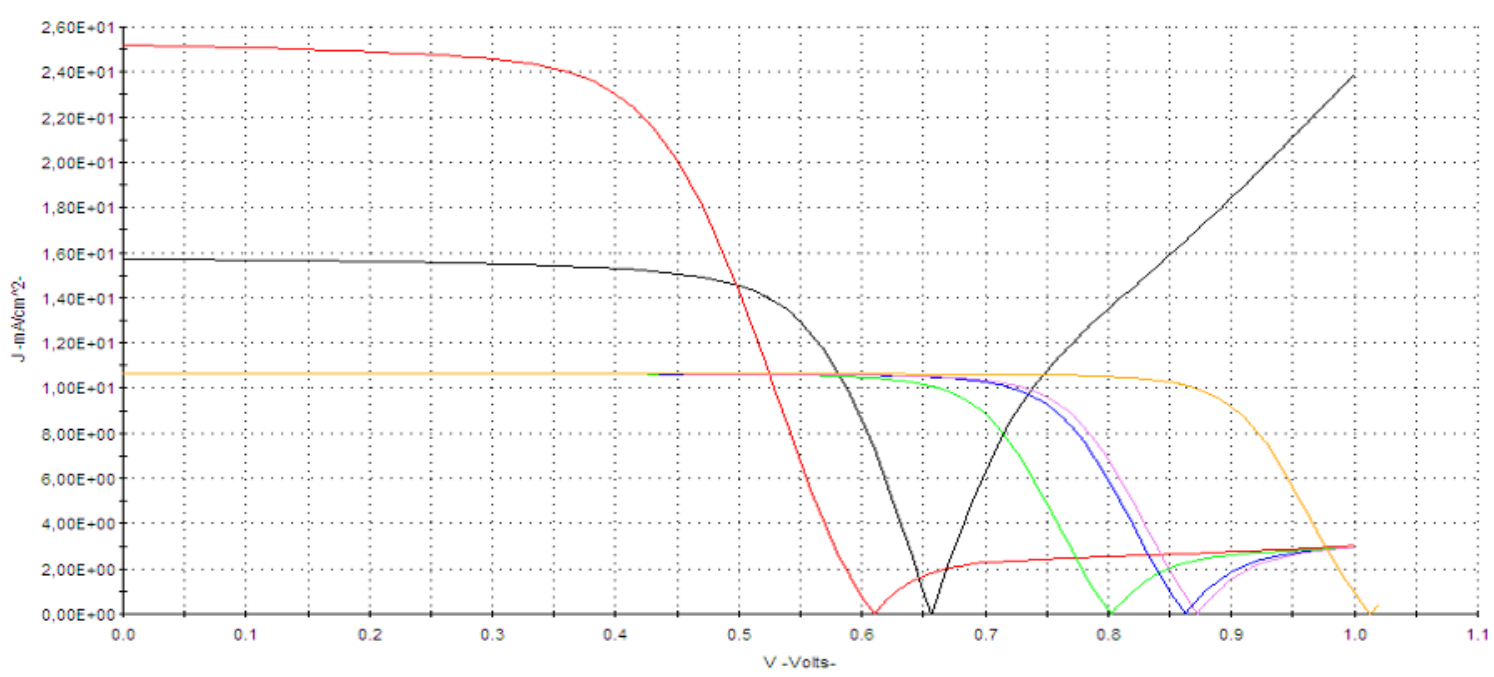

T3BEdotBT3A AMP, Jsc=15.734mA/cm'2; Eff= 7.330\%; FF= 0.710; Voce 0.657

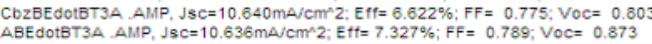

Fig. 5. Simulation by AMPS-1D of the characteristics $\mathrm{J}(\mathrm{V})$ of the organic solar cell based on T3-B-EDOT-B-T3-A/PCBM for different thicknesses. 


\subsection{Effect of the Effective State Density of Electrons and Holes in the Conduction and Valence Bands, Respectively}

We vary the density of effective electron and hole states in the conduction and valence bands, respectively, and set the thickness to $120 \mathrm{~nm}$ and the temperature to $300 \mathrm{k}$. Table 4 shows the results.

For an increase in the effective density, the fill factor increases, the open circuit voltage Voc decreases, and the value of the current density Jsc also decreases. It should also be noted that the increasing the effective density causes a decrease in the value of the PCE; it reaches an optimum value for $10^{21} \mathrm{~cm}^{-3}$.

Table 4. Parameters of the photovoltaic cell for different values of effective electrons and holes densities.

\begin{tabular}{|c|c|c|c|c|}
\hline Density $\left(\mathrm{cm}^{-3}\right)$ & $\begin{array}{c}\text { Jsc(mA } \\
\left.\mathrm{cm} \_2\right)\end{array}$ & Voc(eV) & FF & $\eta \%$ \\
\hline $10^{18}$ & 15.805 & 0.690 & 0.775 & 8.457 \\
\hline $10^{19}$ & 15.778 & 0.703 & 0.740 & 8.209 \\
\hline $10^{20}$ & 15.749 & 0.704 & 0.700 & 7.759 \\
\hline $10^{21}$ & 15.734 & 0.710 & 0.657 & 7.330 \\
\hline $10^{22}$ & 15.725 & 0.720 & 0.609 & 6.903 \\
\hline $10^{23}$ & 15.718 & 0.727 & 0.556 & 6.356 \\
\hline
\end{tabular}

\subsection{Effect of changing donor unit}

In this section, we examined the copolymers studied previously, where the donor unit D1 (T3) was replaced by other donor units (P3, Cbz, F, A) for the first four molecules, and the two donor units D1 and D2 by the donor unit P3 for the last one. We studied the influence of the donor unit substituted in the T3BEdotBT3A copolymer on the cell parameters.
We studied five new copolymers P3BEdotBT3A, CbzBEdotBT3A, FBEdotBT3A, ABEdotBT3A, and P3BEdotBP3A.

In this phase we kept the same parameters used in the previous numerical simulation. We will take the value of thickness equal to $120 \mathrm{~nm}$, set the value temperature at $300 \mathrm{~K}$ and the effective state density of electrons and holes equal to $10^{21} \mathrm{~cm}^{-3}$.

Figure 6 shows the simulation results grouped in Table 5 .

Based on the obtained results, it can be seen that: for the copolymers P3BedotBT3A -PCBM and P3BedotBP3A-PCBM, the values of the PCE are high compared to the other copolymers. On the other hand, the value of the fill factor and the value of the open circuit voltage for P3BedotBP3A, and the value of the current density Jsc for P3BedotBT3A are high compared to the other copolymers.

Therefore, we can conclude that the solar cell becomes more efficient when the $\mathrm{T} 3$ donor is replaced by $\mathrm{P} 3$.

Table 5. Parameters of the photovoltaic cell for different donor units.

\begin{tabular}{|l|l|l|l|l|}
\hline Copolymers & $\begin{array}{l}\text { Jsc(Ma } \\
\mathrm{cm} \_2^{2}\end{array}$ & Voc(V) & FF & $\eta \%$ \\
\hline T3BedotBT3A & 15.734 & 0.710 & 0.657 & 7.330 \\
\hline P3BedotBT3A & 25.186 & 0.605 & 0.610 & 9.295 \\
\hline CbzBEdotBT3A & 10.640 & 0.775 & 0.803 & 6.622 \\
\hline FBEdotBT3A & 10.634 & 0.788 & 0.863 & 7.226 \\
\hline ABEdotBT3A & 10.636 & 0.789 & 0.873 & 7.327 \\
\hline P3BedotBP3A & 10.640 & 0.810 & 1.013 & 8.735 \\
\hline
\end{tabular}

Light IV

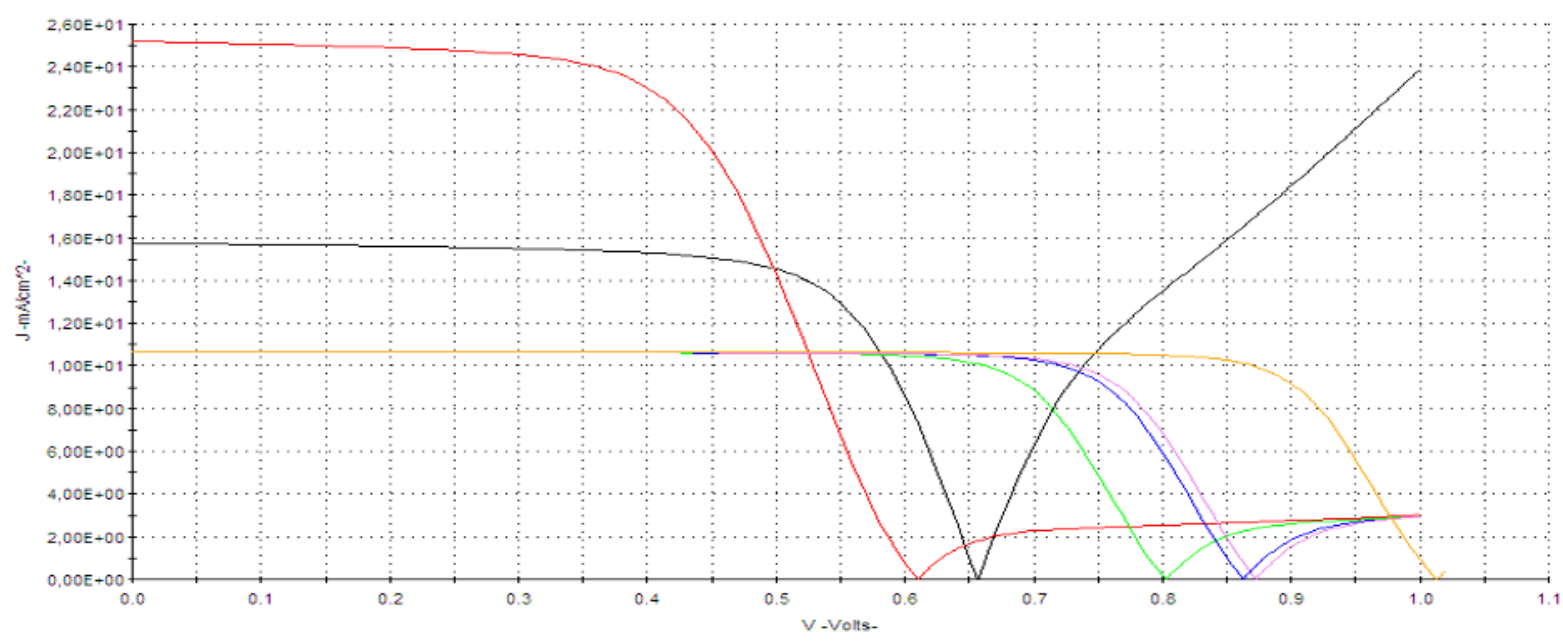

Fig. 6.Simulation by AMPS-1D of the characteristics $J(V)$ of the organic solar cell for different donor units 


\subsection{Effect of incorporating a layer of PEDOT}

Using AMPS-1D software, we obtained the $\mathrm{J}(\mathrm{V})$ characteris tic curves of organic photovoltaic cells based on P3BEdotBT3A / PCBM and P3BEdotBP3A / PCBM in $\mathrm{BHJ}$ structure with a PEDOT layer introduced between the active layer and the anode (figure 7).

In order to identify the effects of the introduced layer, we respectively superimposed these curves with the $J(V)$ characteristic curves of the organic cells based on P3BEdotBT3A / PCBM and P3BEdotBP3A / PCBM obtained previously, respectively (Figures 8 and 9). By analyzing the results, we noticed that the value of shortcircuit current density Jsc decreases for P3BEdotBT3APEDOT and increases for P3BEdotBP3A-PEDOT, but the fill factor varies unlike Jsc for both cells. On the other hand, the value of the open circuit voltage Voc and the efficiency increase.

PCEs of $14.432 \%$ and $15.031 \%$ were obtained from the P3BEdotBT3A and P3BEdotBP3A based devices with the use of PEDOT, which are very important values in photovoltaic conversion. The use of the simulation results indicates an improvement in the conversion efficiency of the organic cell, due to the introduction of PEDOT. The incorporation of the PEDOT layer prevents the diffusion of oxygen and indium from the ITO to the active layer, and promotes the collection of photo-generated positive charges, which improves the performance of the photovoltaic device. The photovoltaic parameters of the simulated organic solar cells are shown in tables 6 and 7 .
Table 6. Parameters of the photovoltaic cell based on P3BEdotBT3A-PCBM before and after the introduction of the PEDOT layer.

\begin{tabular}{|c|c|c|c|c|}
\hline Copolymers & $J_{\text {sc }\left(\mathrm{mAcm}_{\_}\right)}$ & FF & $\begin{array}{l}\text { Voc } \\
(\mathrm{eV})\end{array}$ & $\eta \%$ \\
\hline $\begin{array}{c}\text { P3BEdotBT3A- } \\
\text { PCBM }\end{array}$ & 25.186 & 0.605 & 0.610 & 9.295 \\
\hline $\begin{array}{c}\text { PEDOT- } \\
\begin{array}{c}\text { PEdotBT3A- } \\
\text { PCBM }\end{array}\end{array}$ & 19.902 & 0.622 & 1.166 & 14.432 \\
\hline
\end{tabular}

Table 7. Parameters of the photovoltaic cell based on P3BEdotBP3A-PCBM before and after the introduction of the PEDOT layer

\begin{tabular}{|c|c|c|c|c|}
\hline Copolymers & $\begin{array}{c}\mathrm{Jsc}_{\mathrm{sc}} \mathrm{mA} \\
\left.\mathrm{cm}_{-2}\right)\end{array}$ & FF & $\begin{array}{l}\text { Voc } \\
(\mathrm{eV})\end{array}$ & $\eta \%$ \\
\hline $\begin{array}{c}\text { P3BEdotBP3A- } \\
\text { PCBM }\end{array}$ & 10.640 & 0.810 & 1.013 & 8.735 \\
\hline $\begin{array}{c}\text { PEDOT- } \\
\begin{array}{c}\text { P3BdotBP3A- } \\
\text { PCBM }\end{array}\end{array}$ & 19.773 & 0.621 & 1.225 & 15.031 \\
\hline
\end{tabular}

Al

D1-B-Edot-B-D2-A/PCBM

PEDOT

ITO

Fig. 7. Scheme of the BHJ organic cell based on D1-B-Edot-BD2-A / PCBM with a layer of PEDOT.

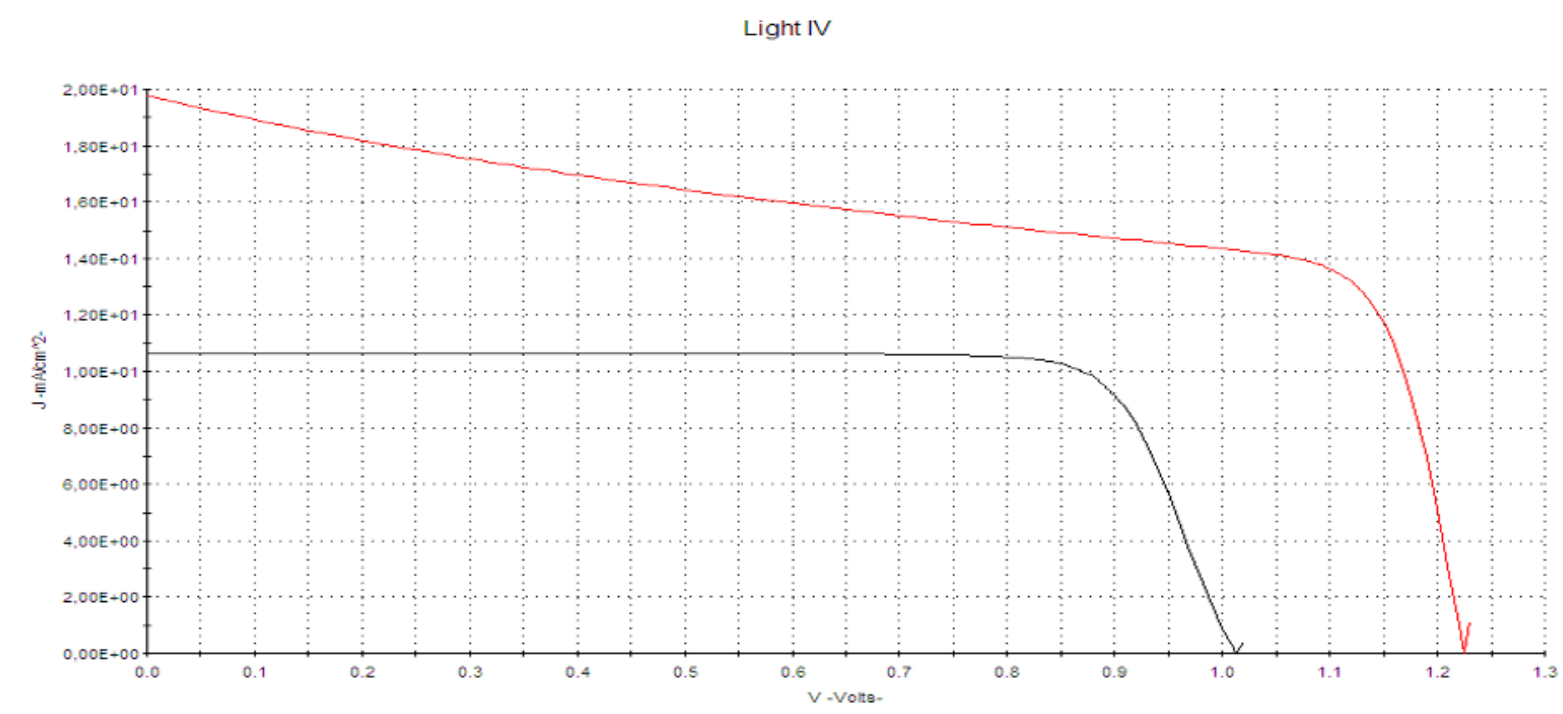

P3BEdotBP3A. AMP, J9c-10.640mA/cm^2; Eff- 8.735\%; FF- 0.810; Voc- 1.013

Fig. 8. Simulation by AMPS-1D of the characteristics J(V) of the organic solar cell based on P3BEdotBT3A- PCBM before and after the incorporation of the film PEDOT 


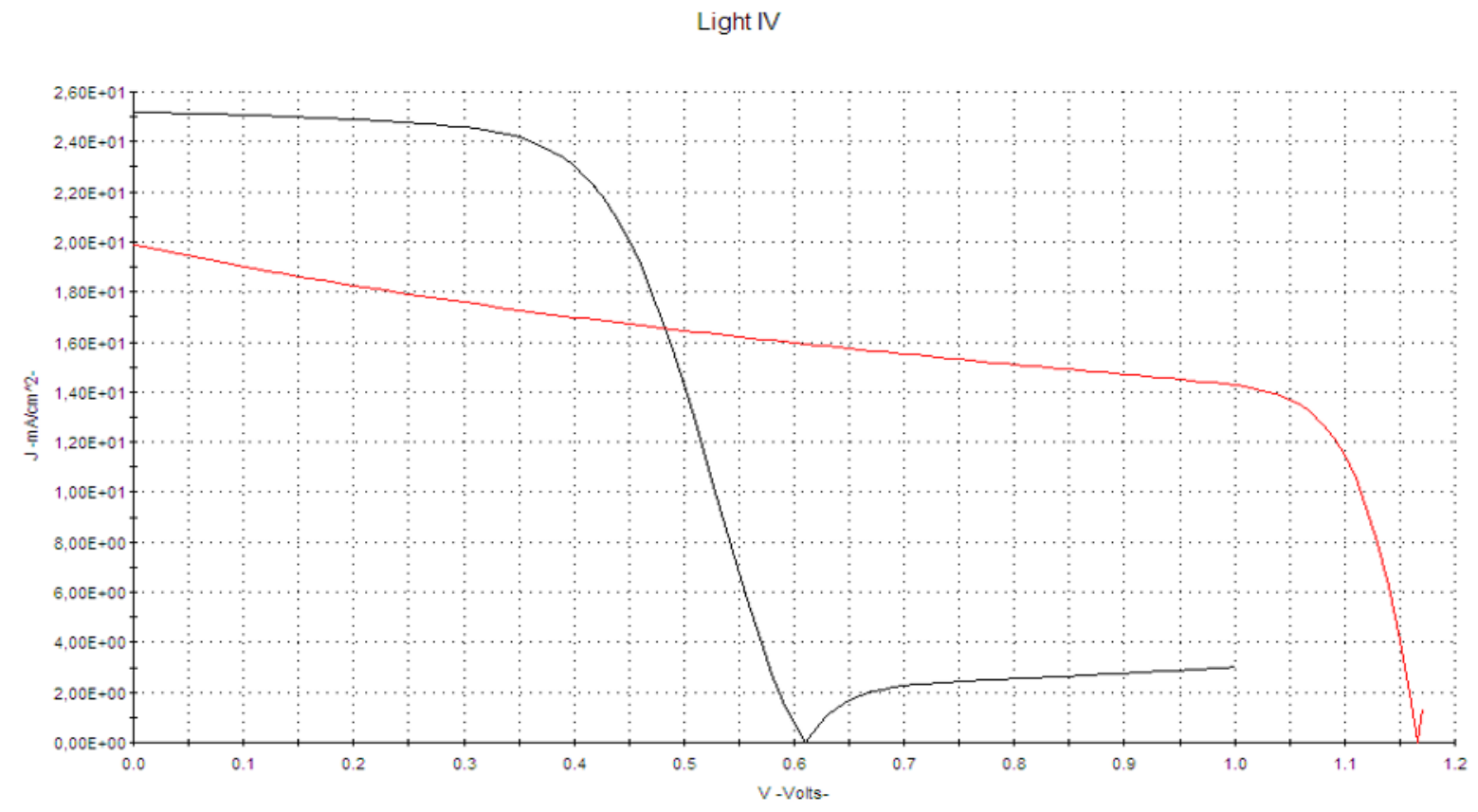

Fig. 9. Simulation by AMPS-1D of the characteristics $J(V)$ of the organic solar cell based on P3BEdotBP3A -PCBM before and after the incorporation of the film PEDOT.

\section{Conclusion}

In summary, we have simulated, the performance of organic solar cells based on D1-BT-EDOT-BT-D2-A/PCBM structures using AMPS-1D program. We have studied the bulk heterojunction structure of the organic solar cell which is composed of T3BedotBT3A / PCBM. We studied the influence of the variation of thickness, temperature, the effective state density of the electrons and holes in the conduction and valence bands respectively, and the effect of changing donor unit on the electrical characteristic of the organic photovoltaic cell. Finally, we have deduced that a PEDOT lay er inserted between the anode and the active layer has a positive effect on the performance of the organic photovoltaic cell. As a result, the Jsc, FF, Voc and PCE values can be improved simultaneously. The P3-B-Edot-BT3-A/PCBM and P3-B-Edot-B-P3-A/PCBM structures delivered an impressive Jsc of $19.902 \mathrm{~mA} / \mathrm{cm} 2$ (19.773), a Voc of $1.166 \mathrm{~V}(1.225)$, an FF of $62.2 \%(62.1 \%)$, and the highest PCE of $14.432 \%(15.031 \%)$ respectively, which represents a clear improvement compared with other results in the literature, such as those of the poly (3-hexylthiophene) (P3HT) -[6,6]-pheny C61 (PCBM) cell and [(Cbz-Mth)-BT]2-PCBM [39].

\section{References}

1. Agence Internationale de l'Energie, World Energy Outlook 2007

2. http://www.iea.org

3. http://www.ademe.fr

4. M.A. Green, K. Emery, Y. Hishikawa et W. Warta, Progress in Photovoltaics: Res earch and Applications 2008, 16,61

5. Z. He, C. Zhong, S. Su, M. Xu, H. Wu and Y. Cao, Nat. Photonics, 2012, 6, 591-595.
6. C. E. Small, S. Chen, J. Subbiah, C. M. Amb, S.-W. Tsang,T.-H. Lai and J. R. Reynolds, Nat. Photonics, 2012, 6, 115-120.

7. Hou, J.; Inganas, O.; Friend, R. H.; Gao, F. Organic solar cells based on non-fullerene acceptors. Nat. Mater. 2018, 17, 119-128.

8. Z-P. Yu, K. Yan, W. Ullah, H. Chen, and CH-Z Li. ACS Appl. Polym. Mater. https://dx.doi.org/10.1021/acsapm.0c00791

9. S-L Chang, K-E Hung, Fong-Yi Cao, K-H Huang, ChSh Hsu, Ch-Yi Liao, Ch-Hao Lee, and Yen-Ju Cheng. ACS Appl. Mater. Interfaces 2019, 11, 33179-33 187. 10.1021/acsami.9b08462.

10. Malki et al., International Journal of Advanced Research in Computer Science and Software Engineering 8(12) ISSN(E): 2277-128X, ISSN(P): 2277-6451, pp. 38-51.

11. Cheng, P.; Li, G.; Zhan, X.; Yang, Y. Next-generation organic photovoltaics based on non-fullerene acceptors. Nat. Photonics 2018, 12, 131-142.

12. Ye, L.; Hu, H.; Ghasemi, M.; Wang, T.; Collins, B. A.; Kim, J.- H.; Jiang, K.; Carpenter, J. H.; Li, H.; Li, Z.; McAfee, T.; Zhao, J.; Chen, X.; Lai, J. L. Y.; Ma, T.; Bredas, J.-L.; Yan, H.; Ade, H. Quantitative relations between interaction parameter, miscibility and function in organic solar cells. Nat. Mater. 2018, 17, 253-260.

13. Zhang, G.; Zhao, J.; Chow, P. C. Y.; Jiang, K.; Zhang, J.; Zhu, Z.; Zhang, J.; Huang, F.; Yan, H. Nonfullerene Acceptor Molecules for Bulk Heterojunction Organic Solar Cells. Chem. Rev. 2018, 118, 3447-3507.

14. Chao, P.; Chen, H.; Zhu, Y.; Lai, H.; Mo, D.; Zheng, N.; Chang, X.; Meng, H.; He, F. A Benzo[1,2-b:4,5 c'] 
Dithiophene-4,8- Dione-Based Polymer Donor Achieving an Efficiency Over 16\%. Adv. Mater. 2020, 32, 1907059

15. Liu, Q.; Jiang, Y.; Jin, K.; Qin, J.; Xu, J.; Li, W.; Xiong, J.; Liu, J.; Xiao, Z.; Sun, K.; Yang, S.; Zhang, X.; Ding, L. 18\% efficiency organic solar cells. Sci. Bull. 2020, 65, 272-275.

16. Yuan, J.; Zhang, Y.; Zhou, L.; Zhang, G.; Yip, H.-L.; Lau, T.-K.; Lu, X.; Zhu, C.; Peng, H.; Johnson, P. A.; Leclerc, M.; Cao, Y.; Ulanski, J.; Li, Y.; Zou, Y. Single-Junction Organic Solar Cell with over $15 \%$ Efficiency Using Fused-Ring Acceptor with ElectronDeficient Core. Joule 2019, 3, 1140-1152.

17. Yu, R.; Yao, H.; Cui, Y.; Hong, L.; He, C.; Hou, J. Improved Charge Transport and Reduced Nonradiative Energy Loss Enable Over 16\% Efficiency in Ternary Polymer Solar Cells. Adv. Mater. 2019, 31, 1902302.

18. Li, S.; Li, C.-Z.; Shi, M.; Chen, H. New Phase for Organic Solar Cell Research: Emergence of Y-Series Electron Acceptors and Their Perspectives. ACS Energy Lett. 2020, 5, 1554-1567.

19. A.J. Attias, Techniques de L'ingénieur, dossierE1862.

20. T. Ilhem, Etude, Mod'elisation, Simulation de cellule solaire organique, Unit'e de Recherche des Matériaux et Energies Renouvelables (URMER), BP 119, 13000 Tlemcen - Algérie, 2018

21.Z. El Malki, A. El karkri, M. Bouachrine, F.S. Spirau3. Springer Nature Switzerland AG 2020, https://doi.org/10.1007/978-3-030-36475-5 28

22. M. Oukachmih, Thèse 2003, Université ToulousePaul Sabatier.

23. C.J. Brabec, A. Cravino, D. Meissner, N.S. Sariciftci, T. Fromherz, M.T. Rispens, L. Sanchez et J.C. Hummelen, Advanced Functional Materials 2001, 11, 374

24. M.C. Scharber, D. Mühlbacher, M. Koppe, P. Denk, C. Waldauf, A.J. Heeger et C.J. Brabec, Advanced Materials 2006, 18, 789;

25. Gunes, S.; Neugebauer, H.; Sariciftci, N. S. J. Chem. Rev. 2006, 107, 1324. [CrossRef] https://doi.org/10.1021/cr050149z

26. Chattopadhyay, D.; Lastella, S.; Kim, S.; Papadimitrakopoulos, F. J. Am. Chem. Soc. 2002, 124, 5, 728. [CrossRef] https://pubs.acs.org/doi/10.1021/ja0172159

27. A. K. K. Kyaw, D. H. Wang, D. Wynands, J. Zhang, T. Q. Nguyen, G. C. Bazan and A. J. Heeger, Improved light harvesting and improved efficiency by insertion of an optical spacer $(\mathrm{ZnO})$ in solution processed small molecule

solar cells, Nano Lett., 2013, 13, 3796-3801.

28. J. Zhou, Y. Zuo, X. Wan, G. Long, Q. Zhang, W. Ni, Y. Liu, Z. Li, G. He, C. Li, B. Kan, M. Li and Y. Chen, Solution processed and high-performance organic solar cells using small molecules with a benzodithiophene unit, J. Am. Chem. Soc., 2013, 135, 8484-8487.

29. B. Kan, Q. Zhang, M. Li, X. Wan, W. Ni, G. Long, Y. Wang, X. Yang, H. Feng and Y. Chen, Solution processed

organic solar cells based on dialkylthiol-substituted benzodithiophene unit with efficiency near $10 \%$, J. Am. Chem. Soc., 2014, 136, 15529-15532.

30. J. Zhou, X. Wan, Y. Liu, Y. Zuo, Z. Li, G. He, G. Long, W. Ni, C. Li, X. Su and Y. Chen, Small molecule based on benzo[1,2- b:4,5-b0] dithiophene unit for high performance solution processed organic solar cells, J. Am. Chem. Soc., 2012, 134, 1634516351.

31. Q. Zhang, B. Kan, F. Liu, G. Long, X. Wan, X. Chen, Y. Zuo, W. Ni, H. Zhang, M. Li, Z. Hu, F. Huang, Y. Cao, Z. Liang, M. Zhang, T. P. Russell and Y. Chen, Small molecule solar cells with efficiency over $9 \%$, Nat. Photonics, 2015, 9, 35-41.

32. B. Kan, M. Li, Q. Zhang, F. Liu, X. Wan, J. Wang, W. Ni, G. Long, X. Yang, H. Feng, Y. Zuo, M. Zhang, F. Huang, Y. Cao, T. P. Russell and Y. A. Chen, Series of simple oligomer-like small molecules based on oligothiophenes for solution processed solar cells with high efficiency, J. Am. Chem. Soc., 2015, 137, 38863893.

33. Y. Liu, C. Chen, Z. Hong, J. Gao, Y. Yang, H. Zhou, L. Dou, G. Li and Y. Yang, Solution processed small molecule solar cells: breaking the $10 \%$ power conversion efficiency, Sci. Rep., 2013, 3, 3356.

34. Heliatek GmbH, Heliatek consolidates its technology leadership by establishing a new world record for organic solartechnology with a cell efficiency of $12 \%$, available at http://www.heliatek.com/wpcontent/uploads/2013/01/ 130116-PR-Heliatekachieves-record-cell-efficiency-forOPV.pdf,January, 2013.

35. V. D. Mihailetchi, J. K. J. van Duren, P. W. M. Blom, J. C. Hummelen, R. A. J. Janssen, J. M. Kroon, M. T.Rispens, W. J. H. Verhees and M. M. Wienk, « Electron transport in a methanofullerene ", Advanced Functional Materials $2003 ; 13(1)$ : 43-46.

36. T. B. Singh, N. Marjanović, G. J. Matt, S. Günes, N. S. Sariciftci, A. Montaigne Ramil, A. Andreev, H. Sitter, R. Schwödiauer and S. Bauer, « High-mobility n-channel organic field-effect transistors based on epitaxially grown C60 films », Organic Electronics $2005 ; 6(3): 105-110$.

37. M. T. Rispens, A. Meetsma, R. Rittberger, C. J. Brabec, N. S. Sariciftci and J. C. Hummelen, " Influence of the solvent on the crystal structure of PCBM and the efficiency of MDMO-PPV:PCBM 'plastic' solar cells », Chemical Communications $2003 ; 17: 2116-2118$.

38. L. M. Popescu, Fullerene based organic solar cells, $\mathrm{PhD}$ thesis, University of Groningen, Netherlands, 2008.

39. A. El Karkri, Z. El Malki, M. Bouachrine, F. SereinSpirauc and J-M Sotiropoulosd. RSC Adv., 2020, 10, 18816. 